\title{
Time differentiated nuclear resonance spectroscopy coupled with pulsed laser heating in diamond anvil cells
}

I. Kupenko, C. Strohm, C. McCammon, V. Cerantola, K. Glazyrin, S. Petitgirard, D. Vasiukov, G. Aprilis, A. I. Chumakov, R. Rüffer, and L. Dubrovinsky

Citation: Review of Scientific Instruments 86, 114501 (2015);

View online: https://doi.org/10.1063/1.4935304

View Table of Contents: http://aip.scitation.org/toc/rsi/86/11

Published by the American Institute of Physics

\section{Articles you may be interested in}

Portable double-sided pulsed laser heating system for time-resolved geoscience and materials science applications

Review of Scientific Instruments 88, 084501 (2017); 10.1063/1.4998985

Melting temperatures of $\mathrm{H}_{2} \mathrm{O}$ up to $72 \mathrm{GPa}$ measured in a diamond anvil cell using $\mathrm{CO}_{2}$ laser heating technique

The Journal of Chemical Physics 140, 074501 (2014); 10.1063/1.4865252

Portable double-sided laser-heating system for Mössbauer spectroscopy and X-ray diffraction experiments at synchrotron facilities with diamond anvil cells

Review of Scientific Instruments 83, 124501 (2012); 10.1063/1.4772458

X-ray diffraction in the pulsed laser heated diamond anvil cell

Review of Scientific Instruments 81, 113902 (2010); 10.1063/1.3499358

New developments in laser-heated diamond anvil cell with in situ synchrotron x-ray diffraction at High Pressure Collaborative Access Team

Review of Scientific Instruments 86, 072201 (2015); 10.1063/1.4926895

BX90: A new diamond anvil cell design for X-ray diffraction and optical measurements

Review of Scientific Instruments 83, 125102 (2012); 10.1063/1.4768541

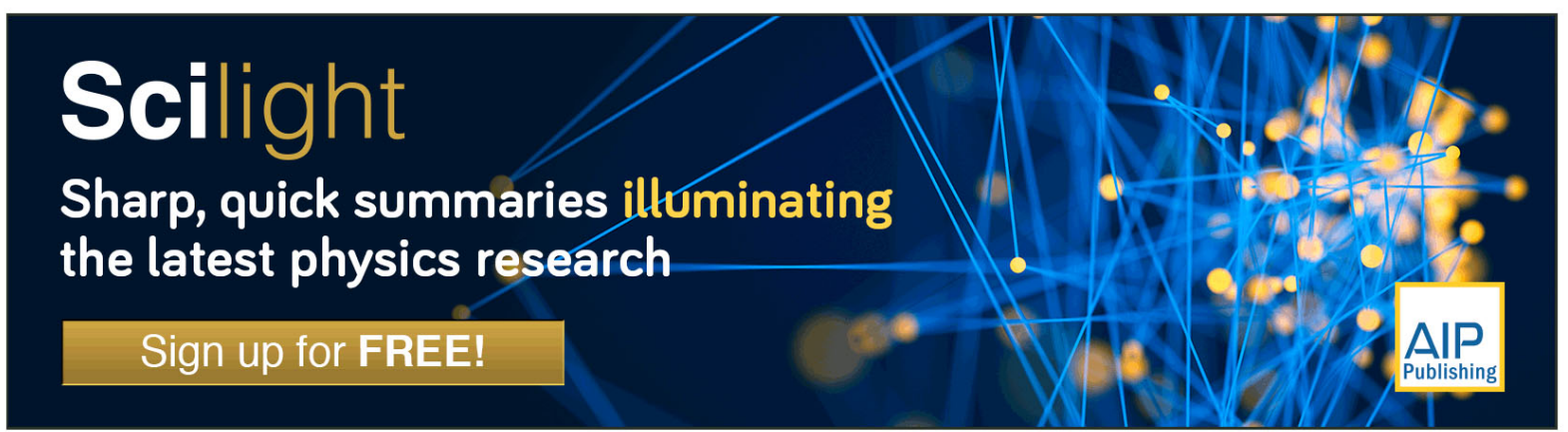




\title{
Time differentiated nuclear resonance spectroscopy coupled with pulsed laser heating in diamond anvil cells
}

\author{
I. Kupenko, ${ }^{1,2, a)}$ C. Strohm, ${ }^{1,2}$ C. McCammon, ${ }^{1}$ V. Cerantola, ${ }^{1}$ K. Glazyrin, ${ }^{3}$ S. Petitgirard, ${ }^{1}$ \\ D. Vasiukov, ${ }^{4}$ G. Aprilis, ${ }^{4}$ A. I. Chumakov, ${ }^{2}$ R. Rüffer ${ }^{2}$ and L. Dubrovinsky ${ }^{1}$ \\ ${ }^{1}$ Bayerisches Geoinstitut, Universität Bayreuth, D-95440 Bayreuth, Germany \\ ${ }^{2}$ ESRF-The European Synchrotron, CS 40220, 38043 Grenoble Cedex 9, France \\ ${ }^{3}$ Photon Science, DESY, D-22607 Hamburg, Germany \\ ${ }^{4}$ Laboratory of Crystallography, Material Physics and Technology at Extreme Conditions, Universität \\ Bayreuth, D-95440 Bayreuth, Germany
}

(Received 18 March 2015; accepted 25 October 2015; published online 11 November 2015)

\begin{abstract}
Developments in pulsed laser heating applied to nuclear resonance techniques are presented together with their applications to studies of geophysically relevant materials. Continuous laser heating in diamond anvil cells is a widely used method to generate extreme temperatures at static high pressure conditions in order to study the structure and properties of materials found in deep planetary interiors. The pulsed laser heating technique has advantages over continuous heating, including prevention of the spreading of heated sample and/or the pressure medium and, thus, a better stability of the heating process. Time differentiated data acquisition coupled with pulsed laser heating in diamond anvil cells was successfully tested at the Nuclear Resonance beamline (ID18) of the European Synchrotron Radiation Facility. We show examples applying the method to investigation of an assemblage containing $\varepsilon$ $\mathrm{Fe}, \mathrm{FeO}$, and $\mathrm{Fe}_{3} \mathrm{C}$ using synchrotron Mössbauer source spectroscopy, $\mathrm{FeCO}_{3}$ using nuclear inelastic scattering, and $\mathrm{Fe}_{2} \mathrm{O}_{3}$ using nuclear forward scattering. These examples demonstrate the applicability of pulsed laser heating in diamond anvil cells to spectroscopic techniques with long data acquisition times, because it enables stable pulsed heating with data collection at specific time intervals that are synchronized with laser pulses. (C) 2015 AIP Publishing LLC. [http://dx.doi.org/10.1063/1.4935304]
\end{abstract}

\section{INTRODUCTION}

Over the last decades, in situ experiments at extreme pressure and temperature conditions have provided many exciting results in the fields of experimental geosciences (Tateno et al., 2010), solid state physics (Eremets et al., 2004), chemistry, and material sciences (Solozhenko et al., 2009). Such achievements only became possible due to advances and developments in synchrotron radiation techniques as well as the evolution of tools for generating extreme conditions. Laser heating in diamond anvil cells (DACs) is a commonly used technique to achieve pressures over $1 \mathrm{Mbar}(100 \mathrm{GPa})$ and temperatures of several thousands of degrees. Many scientific facilities have dedicated stations or beamlines to conduct laser heating in DACs (Prakapenka et al., 2008 and Schultz et al., 2005) that focus on studies of melting (Anzellini et al., 2013), phase transitions (Bykova et al., 2013 and Dubrovinsky et al., 2007), and synthesis of new materials (Salamat et al., 2009). However, laser heating in DACs using continuous wave (CW) solid state lasers, especially at very high temperatures, is often complicated by abrupt changes in the material's emissivity, leading to thermal extinction or flashes, hydrodynamic instability of thin very hot samples, problems with spreading of heated material into or out of the heated area, and possible chemical reactions between sample and thermal insulation and/or diamonds (Dewaele et al., 2010). For these reasons,

\footnotetext{
a) Author to whom correspondence should be addressed. Electronic mail: kupenko@esrf.fr
}

pulsed laser heating offers great advantages as demonstrated recently (Goncharov et al., 2010) to overcome (either partially or fully) the problems mentioned above. Indeed, it is possible to concentrate the energy deposited by each laser pulse into a short time interval and to prevent uncontrolled changes in emissivity due to a low average power. The spreading of material and chemical reactions are minimized due to the very short heating pulses: they allow the thermal insulating material to remain relatively cold, thereby providing more stable and better controlled heating. However, so far pulsed laser heating has been restricted to investigations with short times (dozens of seconds) for data collection, in particular for X-ray diffraction and Raman spectroscopy (Goncharov et al., 2008; Goncharov et al., 2010). Nuclear resonance techniques generally require much longer data collection times and have only been used in combination with continuous heating (Kupenko et al., 2015; Lin et al., 2005; and Zhao et al., 2004), but no work has been reported so far that makes use of pulsed laser heating.

Here, we report for the first time the combination of time differentiated Nuclear Forward Scattering (NFS), Nuclear Inelastic Scattering (NIS), and Synchrotron Mössbauer Source (SMS) spectroscopy with pulsed laser heating in DACs. The approach requires detection schemes that allow synchronization of the laser pulse with nuclear resonance spectroscopic measurements. The schemes can cover an extremely wide range of time scales: from quantum beats with typical periods of nanoseconds, through laser pulses with repetitions on the scale of milliseconds, to typical data acquisition times lasting minutes to hours. We demonstrate that the approach allows 
stable heating during the collection of spectra over several hours and we provide examples of application to studies of geophysically relevant materials.

\section{SCHEME OF THE EXPERIMENT}

NFS, NIS, and SMS spectra were collected at the Nuclear Resonance beamline (ID18) (Rüffer and Chumakov, 1996) of the European Synchrotron Radiation Facility (ESRF). Laser heating was performed using a portable double-sided heating system for DACs (Kupenko et al., 2012) (Fig. 1). The system consists of two lasers which are coupled to the laserheating heads (UniHeads) via optical fibers. The UniHeads combine the functions of laser beam focusing, sample observation, illumination, and optical lens for collection of the light emitted by the sample, further analyzed by spectroradiometry for temperature determination. However, for the NIS and NFS experiments described below, luminescence of $\mathrm{KCl}$ pressure transmitting medium prevented spectroradiometry. We used the SPI50 modulated fiber laser as a laser source. The laser beam was focused to a spot of approximately $50 \mu \mathrm{m}$ in diameter with a flattened intensity profile using a pi-shaper beamexpander (Prakapenka et al., 2008).

The time dependence of the laser pulses was monitored by Thorlabs DET36A/M biased photodiodes (Fig. 1), which detect radiation scattered from a cover glass mounted in the optical path of the laser. The start and duration of the gate signals were adjusted according to the time dependence of the laser pulse (Fig. 2). Previous studies showed that the time scale of heat transfer in DACs is on the order of tens of microseconds (Deemyad et al., 2005 and Goncharov et al., 2010); hence, in order to ensure a stable temperature plateau, we excluded the initial and final $\sim 100 \mu$ s of the laser pulse from the data collection time windows (Fig. 2).

The basis of the acquisition scheme for the experiment was the conventional electronics used for nuclear resonance

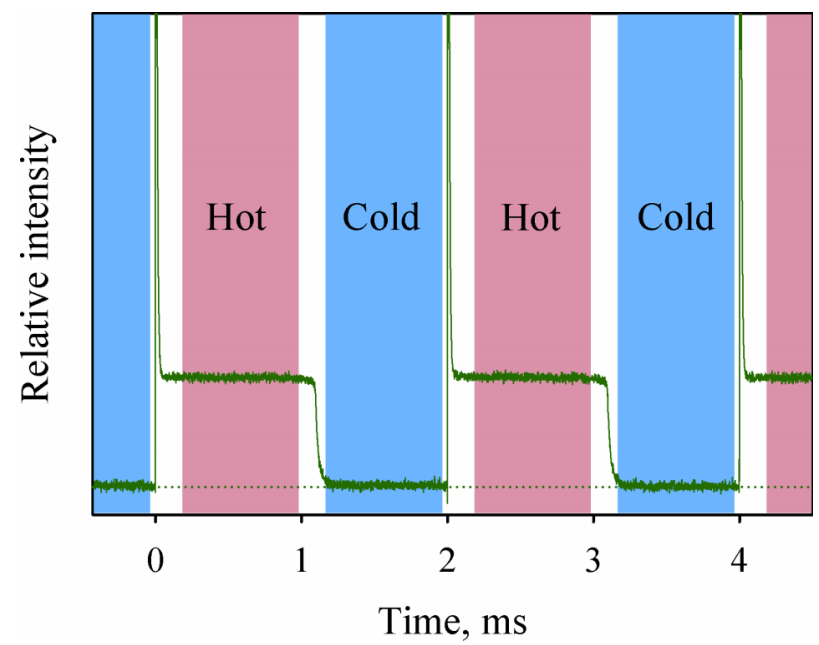

FIG. 2. Timing scheme of the experiment. The time dependence of the laser pulse (green) is monitored with a Thorlabs DET36A/M biased photodiode. Red and blue shadings show the time gates for collection of data from hot and cold samples, respectively.

spectroscopy experiments at the ID18 beamline. The additional electronics for a setup with time differentiated data acquisition is shown in Fig. 3. The impulses of the detectors for NFS, NIS, and SMS were first split using a constant fraction discriminator (CFD) Ortec 935. Additional CFDs were then used to gate the data from hot and cold samples (hereafter referred to as "hot" and "cold" spectra) using the output signals from the delay-generator for the respective time windows. The output signals for "hot" and "cold" spectra were in turn routed to separate counters for NIS and separate multichannel analyzers for NFS and SMS.

For each high pressure experiment, a pressure chamber was made in the Be (NIS) or Re (NFS, SMS) gasket (with an initial diameter of $100 \mu \mathrm{m}$ and a thickness of about $30 \mu \mathrm{m}$ ) with $\mathrm{KCl}$ (NFS, NIS) or Ar (SMS) as a pressure medium

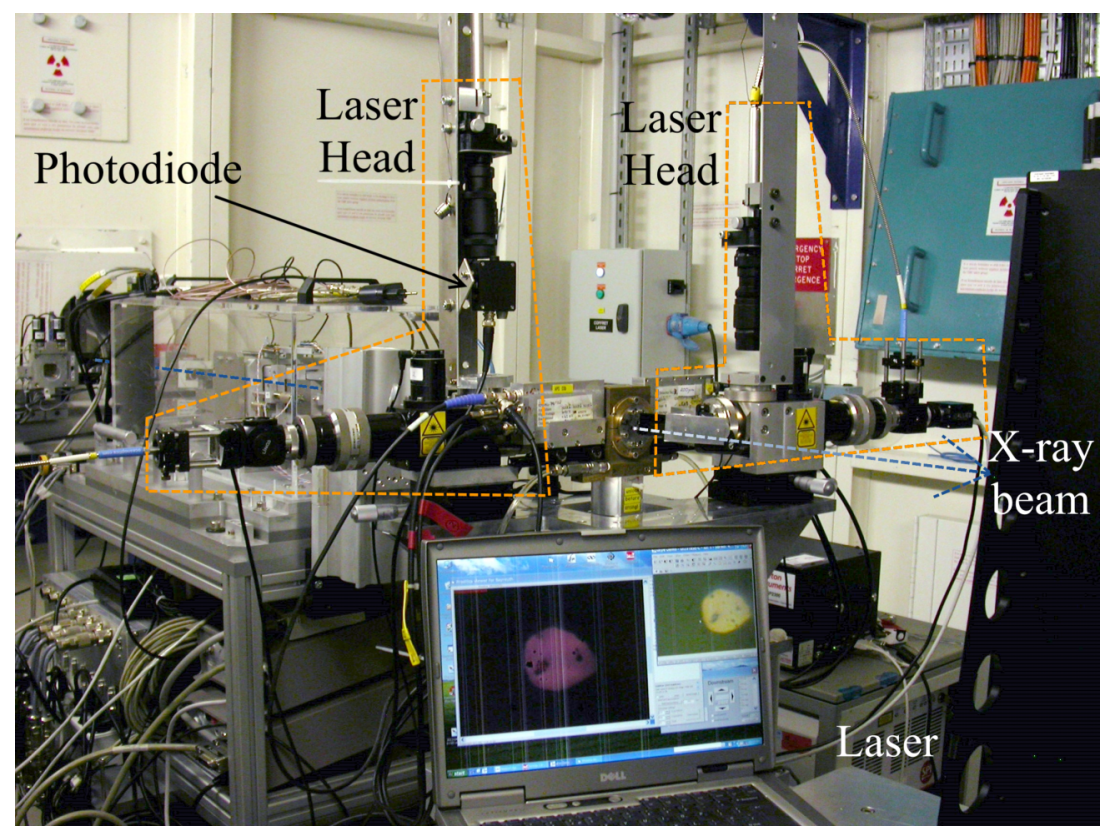

FIG. 1. Double-sided portable laser-heating system mounted for experiments at the Nuclear Resonance beamline (ID18) at the ESRF. 


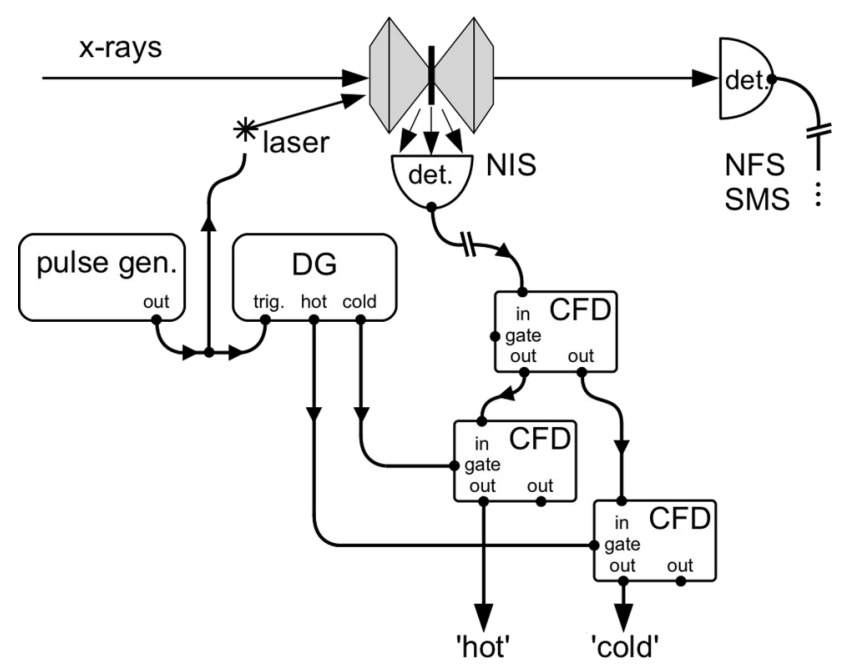

FIG. 3. Acquisition scheme for NIS, NFS, and SMS used for time discriminated data collection coupled with pulsed laser heating in a DAC. Apart from the detectors, only the electronics additional to conventional acquisition schemes is shown. The acquisition of the data for NFS and SMS (not shown) uses a similar scheme as the NIS branch. The output signals "hot" and "cold" are supplied to multi channel analyzers in the case of NFS and SMS and to counters in the case of NIS. Pulse generator: micro-controller-based pulse generator. DG: Stanford DG 535 delay generator. CFD: Ortec 935 constant fraction discriminator.

and a thermal insulator and a small ruby sphere as a pressure marker.

The measurements were performed during operation in "hybrid" mode (NIS) or "multibunch" mode (NFS, SMS) of the storage ring (Characteristics of Beam Modes at the ESRF: http://www.esrf.eu/Accelerators/Operation/Modes). The Xray beam was focused to dimensions of $3.4 \mu \mathrm{m}$ in the vertical and $12 \mu \mathrm{m}$ in the horizontal (NIS), $3.2 \mu \mathrm{m}$ in the vertical and $13.6 \mu \mathrm{m}$ in the horizontal (NFS), or $7 \mu \mathrm{m}$ in the vertical and $14 \mu \mathrm{m}$ in the horizontal (SMS) using multilayer mirrors in Kirkpatrick-Baez geometry.

\section{EXAMPLES}

\section{A. Synchrotron Mössbauer source study of $\varepsilon-\mathrm{Fe}, \mathrm{FeO}$, and $\mathrm{Fe}_{3} \mathrm{C}$}

The recently developed SMS (Potapkin et al., 2012) provides a powerful tool for the study of laser-heated material for geophysical applications. The temperature dependence of the centre shift (CS) in the high-temperature Debye limit is about $\Delta v=-7.3 \times 10^{-4} \mathrm{~mm} / \mathrm{s} \mathrm{K}$ (Maradudin et al., 1962) and, therefore, the difference between the CS values of different components allows measurement of the temperature of the same atoms that are responsible for the absorption (see $\mathrm{Ku}-$ penko et al., 2015). Mössbauer spectroscopy is demanding with regard to the amount of sample; hence, sample thickness is an important parameter. On the other hand, relatively thick samples may introduce temperature gradients in experiments utilizing laser-heated DACs. In order to investigate these trade-offs, we coupled SMS measurements with time-resolved incandescent light spectroradiometry to enable comparison of surface temperatures from spectroradiometry with temperatures determined from changes of the CS. This approach also provided a monitor of temperature variations over long data acquisition times.

A $1 \mu \mathrm{m}$ thick iron foil $95 \%$ enriched in ${ }^{57} \mathrm{Fe}$ was loaded into the pressure chamber at ambient pressure. For high pressure generation, a cylindrical-type BX-90 DAC (Kantor et al., 2012) was used. First, the pressure chamber was compressed to 59(1) GPa and then heated offline by lasers from both sides for $\sim 10 \mathrm{~h}$ at $1850(250) \mathrm{K}$ in order to trigger any possible chemical reactions. Second, the sample was analyzed by Xray diffraction. The diffraction pattern showed diffraction lines indicating the formation of polycrystalline samples of $\mathrm{Fe}_{3} \mathrm{C}$ and $\mathrm{FeO}$ together with a small amount of unreacted $\varepsilon-\mathrm{Fe}$. The appearance of iron oxide can likely be attributed to minor traces of oxygen dissolved in argon, while the source of carbon is from the diamonds.

Finally, the sample was laser heated from both sides using pulses with a time-averaged power of $15 \mathrm{~W}$. The duration of the laser pulses was $1 \mathrm{~ms}$ with a repetition rate of $0.5 \mathrm{kHz}$. SMS spectra of the sample were collected over $30 \mathrm{~min}$ and were fitted using the full transmission integral with a normalized Lorentzian-squared source line shape using the MossA software package (Prescher et al., 2012b). Taking into account the duty cycle due to gating of the measured data results in a net acquisition time of approximately 12 min each for the "hot" and "cold" spectrum.

The collected "hot" and "cold" SMS spectra are presented in Fig. 4. The SMS spectrum of the cold sample can be fitted with a superposition of absorption arising from the three phases identified by $\mathrm{X}$-ray diffraction, namely, $\mathrm{FeO}, \mathrm{Fe}_{3} \mathrm{C}$, and

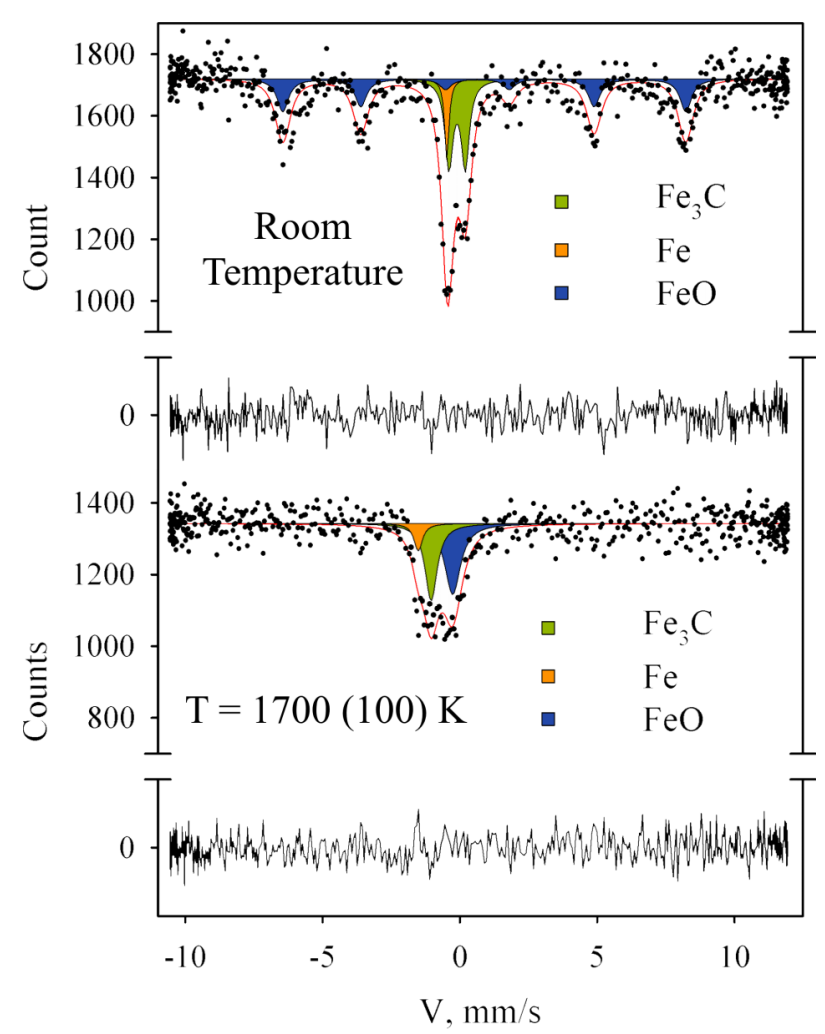

FIG. 4. SMS spectra of $\mathrm{FeO}, \mathrm{Fe}_{3} \mathrm{C}$, and $\varepsilon$-Fe at 59(1) $\mathrm{GPa}$ at the indicated temperatures as determined from the CS difference between cold and hot samples. The solid lines show the theoretical fit and the residuals are indicated below each spectrum. 
TABLE I. Hyperfine parameters of $\mathrm{FeO}, \mathrm{Fe}_{3} \mathrm{C}$, and $\varepsilon$-Fe at $59(1) \mathrm{GPa}$ at the indicated temperatures. CS — centre shift; QS — quadrupole splitting; $B_{h f}-$ hyperfine magnetic field.

\begin{tabular}{llccc}
\hline \hline Temperature & Phase & $\mathrm{CS}^{\mathrm{a}}(\mathrm{mm} / \mathrm{s})$ & $\mathrm{QS}(\mathrm{mm} / \mathrm{s})$ & $B_{h f}(\mathrm{~T})$ \\
\hline \multirow{3}{*}{ Ambient } & $\mathrm{Fe}_{3} \mathrm{C}$ & $-0.10(7)$ & $0.59(11)$ & $\ldots$ \\
& $\mathrm{Fe}$ & $-0.47(13)$ & $\ldots$ & $\ldots$ \\
& $\mathrm{FeO}$ & $0.77(4)$ & $0.25(7)$ & $45.5(3)$ \\
& $\mathrm{Fe}_{3} \mathrm{C}$ & $-1.04(6)$ & $\ldots$ & $\ldots$ \\
$1700(100) \mathrm{K}$ & $\mathrm{Fe}$ & $-1.52(15)$ & $\ldots$ & $\ldots$ \\
& $\mathrm{FeO}$ & $-0.26(6)$ & $\ldots$ & $\ldots$ \\
\hline \hline
\end{tabular}

${ }^{\text {a Relative to } \alpha \text {-Fe. }}$

$\varepsilon$-Fe. The hyperfine parameters of the phases (Table I) are in excellent agreement with the literature values reported for similar pressures (Nasu et al., 1986; Pasternak et al., 1997; and Prescher et al., 2012a). High temperature causes a decrease of the recoil free fraction of resonant absorption of X-rays by nuclei, collapse of the magnetic hyperfine field and quadrupole splitting, and a decrease of CS. The difference between CS of the spectral components from the cold and hot materials $(1.00(8) \mathrm{mm} / \mathrm{s})$ is the same for all three phases within experimental error and confirms heating of the material. The temperature of the hot material determined from the CS difference is 1700(100) K.

The surface temperature of the sample measured using spectroradiometry is shown in Fig. 5. The wavelength range of 640-790 nm was used for fitting the temperature (please see fit examples in Fig. S1 of the supplementary material). Two sets of measurements were performed using an IsoPlane SCT 320 spectrometer with a PI-MAX® 4 1024i ICCD camera from Princeton Instruments. The first set consists of 30 time frames measured with $50 \mu$ s exposure time starting from $200 \mu$ s after a pulse from the delay generator (the pulse is at $0 \mathrm{~ms}$ in Fig. 5). The second set consists of 20 time frames measured with $1 \mu$ s exposure time starting from $90 \mu$ s after the pulse in order to measure the temperature response of the sample at

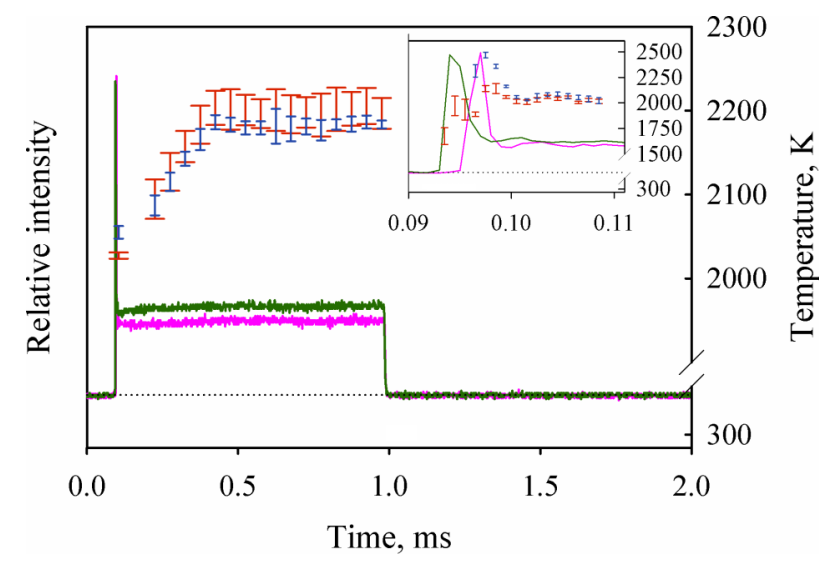

FIG. 5. Sample surface temperature measured using spectroradiometry from upstream (red) and downstream (blue) sides of the DAC. Green and magenta curves show the time dependence of the laser pulse from upstream and downstream sides, respectively. The inset shows an expanded view of time resolved measurements close to the start of laser pulses. Error bars indicate the standard deviations of measurements taken at $\sim 5$ min intervals during a $30 \mathrm{~min}$ heating period. Temperatures not indicated were below the detection limit. the start of the laser spikes. Both sets were measured several times over a 30 min period of heating with $\sim 5$ min intervals between measurements. The standard deviation of derived temperature from measurements performed at different times over a 30 min heating period is less than $100 \mathrm{~K}$. Variations in temperature within the same laser pulse during the time interval used for collection of data from the hot sample (Fig. 2) are also $\sim 100 \mathrm{~K}$. The spectroradiometry measurements therefore confirm the high temperature stability during the SMS measurements.

We find the average temperature of the sample during SMS data collection calculated from the CS difference between cold and hot materials to be lower than the surface temperature measured by spectroradiometry. There are several reasons for this difference. First, the spectroradiometry method is more sensitive to the highest temperature due to the significant temperature dependence of the intensity of emitted thermal radiation. Second, the samples used for the current experiment are opaque; therefore, the incident laser radiation is absorbed in a thin surface layer, resulting in temperature gradients along the heating axis, with a minimum temperature along the axis 5\%-10\% lower than measured (Campbell et al., 2007). Finally, the recoil free fraction becomes smaller with temperature, which suppresses absorption from the hottest parts of the sample and therefore slightly shifts the determination towards lower temperatures in calculating the average temperature from the difference in CS values.

Our results clearly demonstrate that temperatures measured using the commonly used spectroradiometry method should be applied with caution in experiments where laser heated DACs are probed with techniques utilizing a relatively large X-ray beam.

\section{B. Nuclear inelastic scattering study of $\mathrm{FeCO}_{3}$}

NIS is a powerful tool for the study of lattice dynamics that provides a full dynamical and thermodynamic sample characterization (Chumakov and Sturhahn, 1999). Furthermore, it is ideally suited for monitoring of heating processes, because it provides an intrinsic thermometer. The nuclear inelastic absorption may occur with either creation or annihilation of phonons. These processes obey Bose-Einstein statistics, i.e., for any particular energy $E$, the ratio of probabilities for phonon creation (positive part of the spectrum) and phonon annihilation (negative part of the spectrum) is equal to $e^{\frac{|E|}{k_{B} T}}$, where $k_{B}$ is the Boltzmann constant and $T$ is the temperature (see Chumakov and Rüffer, 1998). Therefore, the NIS technique provides a measure of the sample temperature. In order to demonstrate time differentiated NIS coupled with pulsed laser heating in a $\mathrm{DAC}$, we selected $\mathrm{FeCO}_{3}$, the iron end-member of the mineral solid solution magnesite-siderite. This solid solution is currently considered to be one of the most likely carbonate phases to occur in subducting slabs at mantle conditions (Lavina et al., 2009). Carbonates are involved in the Earth's deep carbon cycle through subduction processes; hence, studies of the vibrational, elastic, and thermodynamic properties of these materials are important for interpreting seismological observations on subducted slabs (Merlini et al., 2012). 
Single crystals of $\mathrm{FeCO}_{3}$ with $95 \%{ }^{57} \mathrm{Fe}$ enrichment were synthesized in a multi-anvil apparatus at Bayerisches Geoinstitut at $18 \mathrm{GPa}$ and $1600{ }^{\circ} \mathrm{C}$ from a starting material of ${ }^{57} \mathrm{FeC}_{2} \mathrm{O}_{4}$ powder. The complete protocol of sample synthesis is reported by Cerantola et al. (2015). For high pressure generation, a panoramic DAC constructed at Bayerisches Geoinstitut was used. This type of cell provides a wide opening for detection of the inelastic scattered radiation through the beryllium gasket, perpendicular to the incident beam propagating through the diamond anvils along the cell axis. Several single crystals of ${ }^{57} \mathrm{FeCO}_{3}$ (with dimensions of $\sim 0.01 \times 0.01 \times 0.01 \mathrm{~mm}^{3}$ ) were loaded into the DAC pressure chamber. The pressure chamber was compressed to 25(4) GPa and then heated by the laser from one side with a time-averaged power of $6.2 \mathrm{~W}$. The laser pulse duration was $0.5 \mathrm{~ms}$ with a repetition rate of $1 \mathrm{kHz}$. The energy bandwidth of the high resolution monochromator was $2.3 \mathrm{meV}$. Two avalanche photodiodes were inserted through the panoramic windows of the DAC to collect the inelastic radiation scattered perpendicularly to the synchrotron X-ray beam. The energy dependence of the nuclear inelastic scattering was measured in the energy range -80 to $100 \mathrm{meV}$ around the ${ }^{57} \mathrm{Fe}$ nuclear resonance energy of $14.4 \mathrm{keV}$ with an energy step of $0.2 \mathrm{meV}$. Eight spectra were collected over $40 \mathrm{~min}$ each and then summed to obtain the final spectrum. Taking into account the duty cycle due to gating of the measured data results in a net acquisition time of approximately $16 \mathrm{~min}$ for each "hot" and "cold" spectrum. In order to improve the signal to noise ratio, the final spectrum was binned to a $1 \mathrm{meV}$ grid.

The collected "hot" and "cold" spectra are presented in Fig. 6. Heating causes a decrease of the recoil free fraction of resonant absorption of X-rays by the nuclei. Consequently, the integrated intensity of the NIS spectra (excluding the central elastic peak) increases. The temperatures derived from the detailed balance law for the hot and cold sample are 920(150) $\mathrm{K}$ and 340(30) K, respectively. The derived partial reduced density of phonon states of iron for hot and cold sample is presented in Fig. 7. Extrapolation of the low energy region to zero energy allows a derivation of Debye sound velocities $\left(V_{\mathrm{D}}\right)$ (Achterhold et al., 2002). Combining the derived $V_{\mathrm{D}}$ with the bulk sound velocity enables a calculation

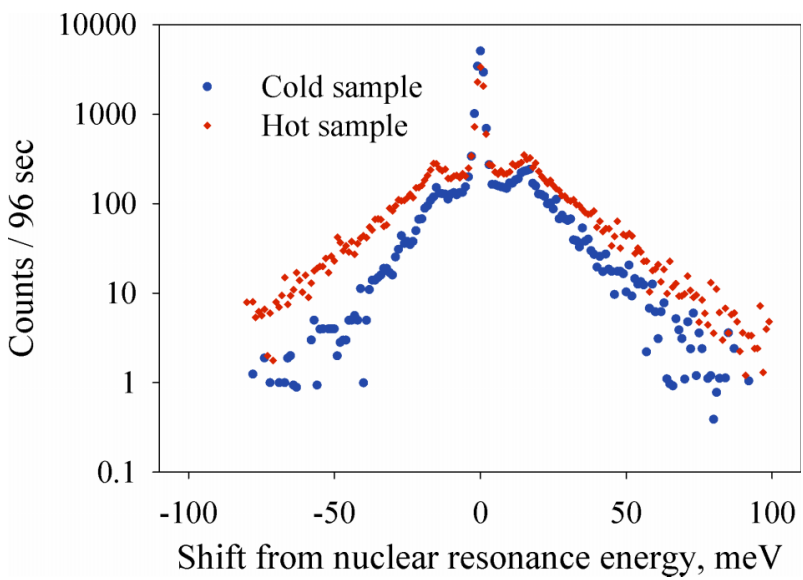

FIG. 6. Energy dependence of nuclear inelastic scattering of ${ }^{57} \mathrm{FeCO}_{3}$ at 25(4) GPa and different temperatures. Cold sample is 340(40) $\mathrm{K}$, while hot sample is 900(140) K. Temperatures were determined from the detailed balance law.
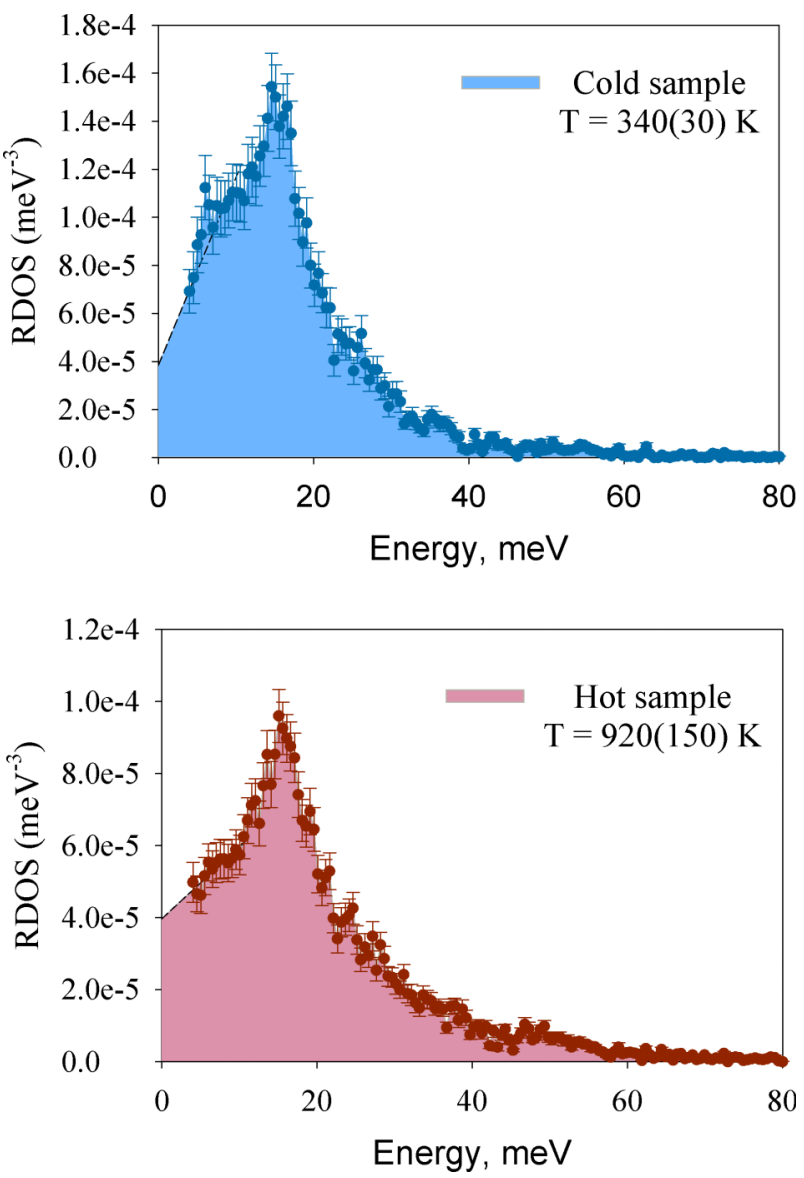

FIG. 7. Partial reduced phonon density of states (RDOS) of iron for $\mathrm{FeCO}_{3}$ at 25(4) GPa and the indicated temperatures. The dashed line shows the extrapolation of the low energy region to zero energy.

of the compressional $\left(V_{\mathrm{p}}\right)$ and shear $\left(V_{\mathrm{s}}\right)$ wave velocities (Lin et al., 2005). For the calculations, we used the thermoelastic parameters of siderite from Litasov et al. (2013). Extracted $V_{\mathrm{D}}, V_{\mathrm{p}}$, and $V_{\mathrm{s}}$ values for $\mathrm{FeCO}_{3}$ are listed in Table II. Sound velocities for the hot and cold samples are indistinguishable within experimental error and are significantly lower than sound velocities for the bulk mantle determined from seismic data (Dziewonski and Anderson, 1981). The results of our study imply that low velocity zones at shallow depths in the lower mantle observed by seismic tomography (Houser et al., 2008) could arise due to iron-bearing carbonates in subducting slabs, regardless of their temperature.

\section{Nuclear forward scattering study of hematite}

At ambient conditions hematite is a rhombohedrally structured wide gap insulator (Hubbard, 1964) with a Néel temperature $\left(T_{\mathrm{N}}\right)$ of $956 \mathrm{~K}$ (van der Woude, 1966). It is one of

TABLE II. Debye $\left(V_{\mathrm{D}}\right)$, compressional $\left(V_{\mathrm{p}}\right)$, and shear $\left(V_{\mathrm{s}}\right)$ wave velocities of $\mathrm{FeCO}_{3}$ at 25(4) $\mathrm{GPa}$ and the indicated temperatures.

\begin{tabular}{llll}
\hline \hline $\mathrm{T}(\mathrm{K})$ & $V_{\mathrm{D}}(\mathrm{km} / \mathrm{s})$ & $V_{\mathrm{p}}(\mathrm{km} / \mathrm{s})$ & $V_{\mathrm{s}}(\mathrm{km} / \mathrm{s})$ \\
\hline $340(30)$ & $4.57(10)$ & $8.12(12)$ & $4.07(8)$ \\
$920(150)$ & $4.52(10)$ & $8.01(13)$ & $4.03(9)$ \\
\hline \hline
\end{tabular}


the dominant phases in banded iron formations (Dobson and Brodholt, 2005) and studies of its properties at high pressure and high temperature are important to understand the fate of subducted banded iron formations. Structural transformations (Bykova et al., 2013; Dubrovinsky et al., 2010; Ito et al., 2009; Liu et al., 2003; and Shim et al., 2009) as well as magnetism and electronic configuration (Badro et al., 2002 and Pasternak et al., 1999) of hematite at high pressures have been intensively investigated over the last decades. However, information on the pressure dependence of $T_{\mathrm{N}}$ (which is relevant to the effect of pressure on electronic structure) is lacking. Here, we report an in situ high-pressure high-temperature investigation of $\alpha$ $\mathrm{Fe}_{2} \mathrm{O}_{3}$ magnetism using NFS.

A cylindrical-type BX-90 DAC (Kantor et al., 2012) was used to generate high pressure. Polycrystalline $\mathrm{Fe}_{2} \mathrm{O}_{3}(\sim 15 \mu \mathrm{m}$ sample thickness) with $95 \%{ }^{57} \mathrm{Fe}$ enrichment was loaded into the pressure chamber. The chamber was compressed to 23(3) GPa and then laser heated from one side with a timeaveraged power of $8 \mathrm{~W}$. The laser pulse duration was $0.5 \mathrm{~ms}$ with a repetition rate of $1 \mathrm{kHz}$. Time spectra of NFS were collected over $30 \mathrm{~min}$ and were fitted using the CONUSS package (Sturhahn, 2000). Taking into account the duty cycle due to gating of the measured data results in a net acquisition time of approximately $12 \mathrm{~min}$ for each "hot" and "cold" spectrum.

The time spectrum of NFS of the cold sample (Fig. 8(a)) shows high frequency quantum beats arising from magnetic hyperfine interactions. It can be fitted with a single component with a magnetic hyperfine field $\left(B_{h f}\right)$ of 51.4(1) T, consistent with previously reported values (Pasternak et al., 1999 and Shim et al., 2009). The spectrum of the sample at high temperature (Fig. 8(b)) shows a more complicated pattern that can be fitted by two magnetic components: one with $B_{h f}$ equal to 51.7(2) T and the second with $B_{h f}$ equal to 39.0(3) T. $B_{h f}$ of the first component is the same as that for the component from the "cold" spectrum, and, therefore, is considered to correspond to material that remained unheated due to one-sided heating with low power, while the second component corresponds to the hot material. The spectrum was fitted assuming that the scattering arising from hot and cold material is coherent, which means an axial temperature gradient in the DAC. The fraction of the hot material within the X-ray beam is predominant: its relative contribution is 55(3)\%, even without consideration of the temperature effect on the recoil free fraction, which reduces with temperature (Gütlich et al., 2011).

The CS of different components corresponding to different parts of the sample enables determination of temperature in those parts of the sample (see Section III A). Based on the CS difference between low- $B_{h f}$ and high- $B_{h f}$ components $(0.83(4) \mathrm{mm} / \mathrm{s})$ from the "hot" spectrum, we determined the temperature of the hot material to be 1400(70) K. The component assigned to hot material is still magnetically ordered, indicating that $T_{\mathrm{N}}$ of $\mathrm{Fe}_{2} \mathrm{O}_{3}$ increases with pressure to well above $1400 \mathrm{~K}$. The phase boundary of the transition of corundumstructured $\alpha-\mathrm{Fe}_{2} \mathrm{O}_{3}$ into a $\mathrm{Rh}_{2} \mathrm{O}_{3}$-II type phase (Ito et al., 2009) crosses the geotherm (Katsura et al., 2010) at $18 \mathrm{GPa}$ and $1900 \mathrm{~K}$. For colder subduction slabs, the transition pressure is even higher. While a detailed pressure dependence of $T_{\mathrm{N}}$ of $\alpha-\mathrm{Fe}_{2} \mathrm{O}_{3}$ remains to be investigated, our observation of magnetically ordered hematite at $23 \mathrm{GPa}$ and $1400 \mathrm{~K}$
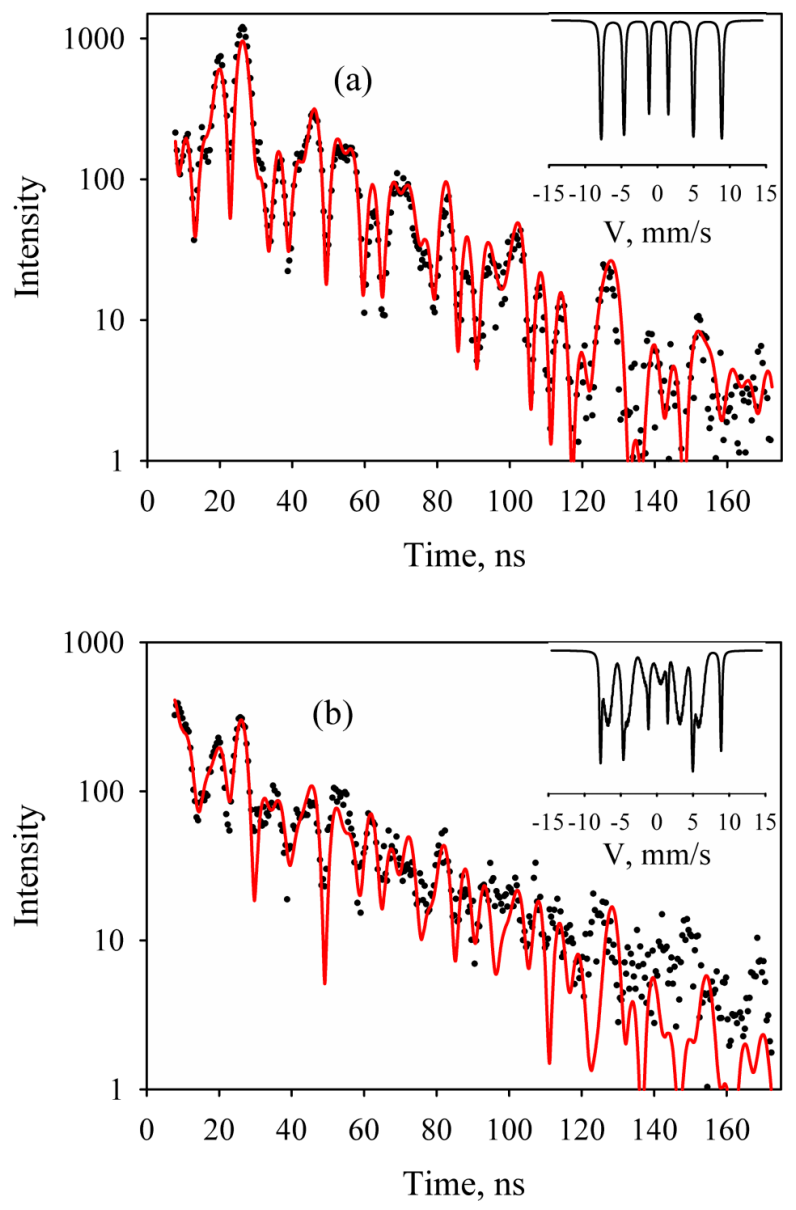

FIG. 8. Time spectra of nuclear forward scattering of $\mathrm{Fe}_{2} \mathrm{O}_{3}$ at 23(3) GPa at (a) room temperature and (b) $\mathrm{T}=1400(70) \mathrm{K}$. The temperature was determined from the difference between the CS values of the two components. The solid lines show the theoretical fits, while the insets show the corresponding energy spectra calculated from the fits.

demonstrates that it could play a role in magnetism of the Earth's deep interior.

\section{CONCLUSION}

We have shown the feasibility of time differentiated measurements coupled with pulsed laser heating in DACs for experiments with long data acquisition times. We presented examples of application of the method to in situ studies of geophysically relevant materials using nuclear resonance spectroscopy. We showed that temperatures measured by the commonly used spectroradiometry method could be notably higher than the bulk temperature of the sample probed by a relatively large X-ray beam. We derived sound velocities of $\mathrm{FeCO}_{3}$ and showed that iron-bearing carbonates could contribute to low velocity zones in the shallow part of the lower mantle. We observed that the Néel temperature of $\mathrm{Fe}_{2} \mathrm{O}_{3}$ increases to a value well above $1400 \mathrm{~K}$ at $23 \mathrm{GPa}$, suggesting its possible role in magnetism at depths of the transition zone and the shallow lower mantle. The possibility to use time gates for the collection of spectra from the sample during pulsed heating enables the same part of the sample to be probed in both the hot and cold state. Pulsed heating reduces the risk of undesirable chemical reactions, and thus provides 
improved stability of heating. The approach can be applied to other laser-pump-probe experiments with X-ray techniques requiring long acquisition times, for example, $\mathrm{X}$-ray emission and X-ray absorption spectroscopy.

\section{ACKNOWLEDGMENTS}

We acknowledge the European Synchrotron Radiation Facility for provision of synchrotron radiation facilities (ID18) and S. Linhardt (Bayerisches Geoinstitut) for help with the preparation of electronic components. The work was partly supported by the German Federal Ministry of Education and Research (BMBF), the German Research Foundation (DFG), and the PROCOPE exchange program.

Achterhold, K., Keppler, C., Ostermann, A., Van Bürck, U., Sturhahn, W., Alp, E. E., and Parak, F. G., "Vibrational dynamics of myoglobin determined by the phonon-assisted Mössbauer effect," Phys. Rev. E 65, 051916 (2002).

Anzellini, S., Dewaele, A., Mezouar, M., Loubeyre, P., and Morard, G., "Melting of iron at Earth's inner core boundary based on fast X-ray diffraction," Science 340, 464-466 (2013).

Badro, J., Fiquet, G., Struzhkin, V. V., Somayazulu, M., Mao, H., Shen, G., and Le Bihan, T., "Nature of the high-pressure transition in $\mathrm{Fe}_{2} \mathrm{O}_{3}$ hematite," Phys. Rev. Lett. 89, 205504 (2002).

Bykova, E., Bykov, M., Prakapenka, V., Konôpková, Z., Liermann, H.-P., Dubrovinskaia, N., and Dubrovinsky, L., "Novel high pressure monoclinic $\mathrm{Fe}_{2} \mathrm{O}_{3}$ polymorph revealed by single-crystal synchrotron X-ray diffraction studies," High Pressure Res. 33, 534-545 (2013).

Campbell, A. J., Seagle, C. T., Heinz, D. L., Shen, G., and Prakapenka, V. B., "Partial melting in the iron-sulfur system at high pressure: A synchrotron X-ray diffraction study," Phys. Earth Planet. Inter. 162, 119-128 (2007).

Cerantola, V., McCammon, C. A., Kupenko, I., Kantor, I. Y., Marini, C., Kantor, A., Wilke, A., Solopova, N., Pascarelli, S., and Dubrovinsky, L. S., "High-pressure spectroscopic study of siderite $\left(\mathrm{FeCO}_{3}\right)$ with focus on spin crossover," Am. Mineral. (to be published).

Chumakov, A. and Rüffer, R., "Nuclear inelastic scattering," Hyperfine Interact. 113, 59-79 (1998).

Chumakov, A. I. and Sturhahn, W., "Experimental aspects of inelastic nuclear resonance scattering," Hyperfine Interact. 123-124, 781-808 (1999).

Deemyad, S., Sterer, E., Barthel, C., Rekhi, S., Tempere, J., and Silvera, I. F., "Pulsed laser heating and temperature determination in a diamond anvil cell,” Rev. Sci. Instrum. 76, 1-4 (2005).

Dewaele, A., Mezouar, M., Guignot, N., and Loubeyre, P., "High melting points of tantalum in a laser-heated diamond anvil cell," Phys. Rev. Lett. 104, 255701 (2010).

Dobson, D. P. and Brodholt, J. P., "Subducted banded iron formations as a source of ultralow-velocity zones at the core-mantle boundary," Nature 434, 371-374 (2005).

Dubrovinsky, L., Boffa-Ballaran, T., Glazyrin, K., Kurnosov, A., Frost, D., Merlini, M., Hanfland, M., Prakapenka, V. B., Schouwink, P., Pippinger, T., and Dubrovinskaia, N., "Single-crystal X-ray diffraction at megabar pressures and temperatures of thousands of degrees," High Pressure Res. 30, 620-633 (2010).

Dubrovinsky, L., Dubrovinskaia, N., Narygina, O., Kantor, I., Kuznetzov, A., Prakapenka, V. B., Vitos, L., Johansson, B., Mikhaylushkin, A. S., Simak, S. I., and Abrikosov, I. A., "Body-centered cubic iron-nickel alloy in Earth's core," Science 316, 1880-1883 (2007).

Dziewonski, A. M. and Anderson, D. L., "Preliminary reference Earth model," Phys. Earth Planet. Inter. 25, 297-356 (1981).

Eremets, M. I., Gavriliuk, A. G., Trojan, I. A., Dzivenko, D. A., and Boehler, R., "Single-bonded cubic form of nitrogen," Nat. Mater. 3, 558-563 (2004).

Goncharov, A., Crowhurst, J., Struzhkin, V., and Hemley, R., "Triple point on the melting curve and polymorphism of nitrogen at high pressure," Phys. Rev. Lett. 101, 095502 (2008).

Goncharov, A. F., Prakapenka, V. B., Struzhkin, V. V., Kantor, I., Rivers, M. L., and Dalton, D. A., "X-ray diffraction in the pulsed laser heated diamond anvil cell," Rev. Sci. Instrum. 81, 113902 (2010).

Gütlich, P., Bill, E., and Trautwein, A. X., Mössbauer Spectroscopy and Transition Metal Chemistry (Springer, Berlin, Heidelberg, 2011).
Houser, C., Masters, G., Shearer, P., and Laske, G., "Shear and compressional velocity models of the mantle from cluster analysis of long-period waveforms," Geophys. J. Int. 174, 195-212 (2008).

Hubbard, J., "Electron correlations in narrow energy bands. II. The degenerate band case," Proc. R. Soc. A 277, 237-259 (1964).

Ito, E., Fukui, H., Katsura, T., Yamazaki, D., Yoshino, T., Aizawa, Y., Kubo, A., Yokoshi, S., Kawabe, K., Zhai, S., Shatzkiy, A., Okube, M., Nozawa, A., and Funakoshi, K.-I., "Determination of high-pressure phase equilibria of $\mathrm{Fe}_{2} \mathrm{O}_{3}$ using the Kawai-type apparatus equipped with sintered diamond anvils," Am. Mineral. 94, 205-209 (2009).

Kantor, I., Prakapenka, V., Kantor, A., Dera, P., Kurnosov, A., Sinogeikin, S., Dubrovinskaia, N., and Dubrovinsky, L., "BX90: A new diamond anvil cell design for X-ray diffraction and optical measurements," Rev. Sci. Instrum. 83, 125102 (2012).

Katsura, T., Yoneda, A., Yamazaki, D., Yoshino, T., and Ito, E., "Adiabatic temperature profile in the mantle," Phys. Earth Planet. Inter. 183, 212-218 (2010)

Kupenko, I., Dubrovinsky, L., Dubrovinskaia, N., McCammon, C. A., Glazyrin, K., Bykova, E., Ballaran, T. B., Sinmyo, R., Chumakov, A. I., Potapkin, V., Kantor, A., Rüffer, R., Hanfland, M., Crichton, W., and Merlini, M., "Portable double-sided laser-heating system for Mössbauer spectroscopy and X-ray diffraction experiments at synchrotron facilities with diamond anvil cells," Rev. Sci. Instrum. 83, 124501 (2012).

Kupenko, I., McCammon, C. A., Sinmyo, R., Cerantola, V., Potapkin, V., Chumakov, A. I., Kantor, A., Rüffer, R., and Dubrovinsky, L., "Oxidation state of the lower mantle: In situ observations of the iron electronic configuration in bridgmanite at extreme conditions," Earth Planet. Sci. Lett. 423, 78-86 (2015).

Lavina, B., Dera, P., Downs, R. T., Prakapenka, V., Rivers, M., Sutton, S., and Nicol, M., "Siderite at lower mantle conditions and the effects of the pressure-induced spin-pairing transition," Geophys. Res. Lett. 36, L23306, doi:10.1029/2009GL039652 (2009).

Lin, J.-F., Sturhahn, W., Zhao, J., Shen, G., Mao, H.-K., and Hemley, R. J., "Sound velocities of hot dense iron: Birch's law revisited," Science $\mathbf{3 0 8}$ 1892-1894 (2005).

Litasov, K. D., Shatskiy, A., Gavryushkin, P. N., Sharygin, I. S., Dorogokupets, P. I., Dymshits, A. M., Ohtani, E., Higo, Y., and Funakoshi, K., "P-V-T equation of state of siderite to 33GPa and 1673K," Phys. Earth Planet. Inter. 224, 83-87 (2013).

Liu, H., Caldwell, W. A., Benedetti, L. R., Panero, W., and Jeanloz, R., "Static compression of $\alpha-\mathrm{Fe}_{2} \mathrm{O}_{3}$ : Linear incompressibility of lattice parameters and high-pressure transformations," Phys. Chem. Miner. 30, 582-588 (2003).

Maradudin, A. A., Flinn, P. A., and Ruby, S., "Velocity shift of the Mössbauer resonance," Phys. Rev. 126, 9-23 (1962).

Merlini, M., Crichton, W. A., Hanfland, M., Gemmi, M., Müller, H., Kupenko, I., and Dubrovinsky, L., "Structures of dolomite at ultrahigh pressure and their influence on the deep carbon cycle," Proc. Natl. Acad. Sci. U. S. A. 109, 13509-13514 (2012).

Nasu, S., Kurimoto, K., Nagatomo, S., Endo, S., and Fujita, F. E., “" ${ }^{57} \mathrm{Fe}$ Mössbauer study under high pressure; $\varepsilon$ - $\mathrm{Fe}$ and $\mathrm{Fe}_{2} \mathrm{O}_{3}$," Hyperfine Interact. 29, 1583-1586 (1986).

Pasternak, M., Taylor, R., Jeanloz, R., Li, X., Nguyen, J., and McCammon, C., "High pressure collapse of magnetism in $\mathrm{Fe}_{0.94} \mathrm{O}$ : Mössbauer spectroscopy beyond 100 GPa," Phys. Rev. Lett. 79, 5046-5049 (1997).

Pasternak, M. P., Rozenberg, G. K., Machavariani, G. Y., Naaman, O., Taylor, R. D., and Jeanloz, R., "Breakdown of the Mott-Hubbard state in $\mathrm{Fe}_{2} \mathrm{O}_{3}$ : A first-order insulator-metal transition with collapse of magnetism at 50 GPa," Phys. Rev. Lett. 82, 4663-4666 (1999).

Potapkin, V., Chumakov, A. I., Smirnov, G. V., Celse, J. P., Rüffer, R., McCammon, C., and Dubrovinsky, L., "The ${ }^{57} \mathrm{Fe}$ synchrotron Mössbauer source at the ESRF," J. Synchrotron Radiat. 19, 559-569 (2012).

Prakapenka, V. B., Kubo, A., Kuznetsov, A., Laskin, A., Shkurikhin, O., Dera, P., Rivers, M. L., and Sutton, S. R., "Advanced flat top laser heating system for high pressure research at GSECARS: Application to the melting behavior of germanium," High Pressure Res. 28, 225-235 (2008).

Prescher, C., Dubrovinsky, L., McCammon, C., Glazyrin, K., Nakajima, Y., Kantor, A., Merlini, M., and Hanfland, M., "Structurally hidden magnetic transitions in $\mathrm{Fe}_{3} \mathrm{C}$ at high pressures," Phys. Rev. B 85, 140402 (2012a).

Prescher, C., McCammon, C., and Dubrovinsky, L., "MossA: A program for analyzing energy-domain Mössbauer spectra from conventional and synchrotron sources," J. Appl. Crystallogr. 45, 329-331 (2012b).

Rüffer, R. and Chumakov, A. I., "Nuclear resonance beamline at ESRF," Hyperfine Interact. 97-98, 589-604 (1996). 
Salamat, A., Woodhead, K., McMillan, P., Cabrera, R., Rahman, A., Adriaens, D., Corà, F., and Perrillat, J.-P., "Tetrahedrally bonded dense $\mathrm{C}_{2} \mathrm{~N}_{3} \mathrm{H}$ with a defective wurtzite structure: X-ray diffraction and Raman scattering results at high pressure and ambient conditions," Phys. Rev. B 80, 104106 (2009).

Schultz, E., Mezouar, M., Crichton, W., Bauchau, S., Blattmann, G., Andrault, D., Fiquet, G., Boehler, R., Rambert, N., Sitaud, B., and Loubeyre, P., "Double-sided laser heating system for in situ high pressure-high temperature monochromatic x-ray diffraction at the ESRF," High Pressure Res. 25, 71-83 (2005).

See supplementary material at http://dx.doi.org/10.1063/1.4935304 for fit examples.

Shim, S.-H., Bengtson, A., Morgan, D., Sturhahn, W., Catalli, K., Zhao, J., Lerche, M., and Prakapenka, V., "Electronic and magnetic structures of the postperovskite-type $\mathrm{Fe}_{2} \mathrm{O}_{3}$ and implications for planetary magnetic records and deep interiors," Proc. Natl. Acad. Sci. U. S. A. 106, 5508-5512 (2009).

Solozhenko, V., Kurakevych, O., Andrault, D., Le Godec, Y., and Mezouar, M., "Ultimate metastable solubility of boron in diamond: Synthesis of superhard diamondlike BC $_{5}$," Phys. Rev. Lett. 102, 015506 (2009).

Sturhahn, W., "CONUSS and PHOENIX: Evaluation of nuclear resonant scattering data," Hyperfine Interact. 125, 149-172 (2000).

Tateno, S., Hirose, K., Ohishi, Y., and Tatsumi, Y., "The structure of iron in Earth's inner core," Science 330, 359-361 (2010).

van der Woude, F., "Mössbauer effect in $\alpha-\mathrm{Fe}_{2} \mathrm{O}_{3}$," Phys. Status Solidi B 17, 417-432 (1966).

Zhao, J., Sturhahn, W., Lin, J., Shen, G., Alp, E. E., and Mao, H., "Nuclear resonant scattering at high pressure and high temperature," High Pressure Res. 24, 447-457 (2004). 